\title{
Angiostrongylus vasorum beim Hund
}

\author{
Anton Heusinger
}

Die Infektion mit Angiostrongylus vasorum wurde erstmals im 19. Jahrhundert in Südfrankreich beschrieben (deshalb „französischer Herzwurm“). Seither stammen Berichte darüber aus allen möglichen Regionen der Welt - häufiger vom Fuchs, seltener vom Hund. In Deutschland ist der Befall mit diesem Lungenwurm beim Hund erst in letzter Zeit vermehrt in den einer Infektion gravierend sein können, müssen gezielte Untersuchungen durchgeführt werden.

\section{Lebenszyklus}

Infizierte Hunde oder Füchse scheiden Larven 1 mit dem Kot aus. Diese werden von Schnecken (Zwischenwirten) aufgenommen und entwickeln sich in den Schnecken nach 2 Häutungen zu infektiösen Larven 3. Die Hunde stecken sich durch den Verzehr infizierter Schnecken an. Dies kann natürlich auch passieren, wenn sie zusammen mit Gras oder Obst die Schnecken aufnehmen. Im Hund verlassen die Larven den Darm und gelangen zunächst in die Mesenteriallymphknoten. Von dort wandern sie zur Pulmonalarterie oder zur rechten Herzseite ungefähr 10 Tage nach der Infektion. Geschlechtsreife weibliche Würmer setzen Eier ab, die in die Gefäße der Lunge Fokus gerückt. Da die klinischen Folgen

gelangen. Dort entwickeln sich die Larven, die nach dem Schlupf die Alveolen erreichen. Von hier werden sie hochgehustet, mit dem Schleim abgeschluckt und schließlich mit dem Kot ausgeschieden.

Die Präpatenz beträgt 33-35 Tage, die adulten Würmer können im Hund für Jahre verbleiben, wenn sie nicht mit einem geeigneten Wurmmittel abgetrieben werden.

Die Infektion mit Angiostrongylus vasorum ist keine Zoonose und kann nicht direkt von Hund zu Hund übertragen werden.

\section{Klinische Symptome}

Die klinischen Symptome sind sehr breit gefächert und treten auf, wenn die Larven die Pulmonalarterie erreicht haben. Sobald die weiblichen Würmer mit der Eiablage beginnen, verschärfen sich in der Regel die Symptome durch Entzündungsvorgänge in der Lunge und den Lungengefäßen.

Die akute Symptomatik umfasst:

- trockener, harter Husten

- Anorexie

- allgemeine Abgeschlagenheit

- ggf. Anämie

- ggf. Gerinnungsstörungen mit ihren Folgen (Meläna, Hämoptysis, verzö-

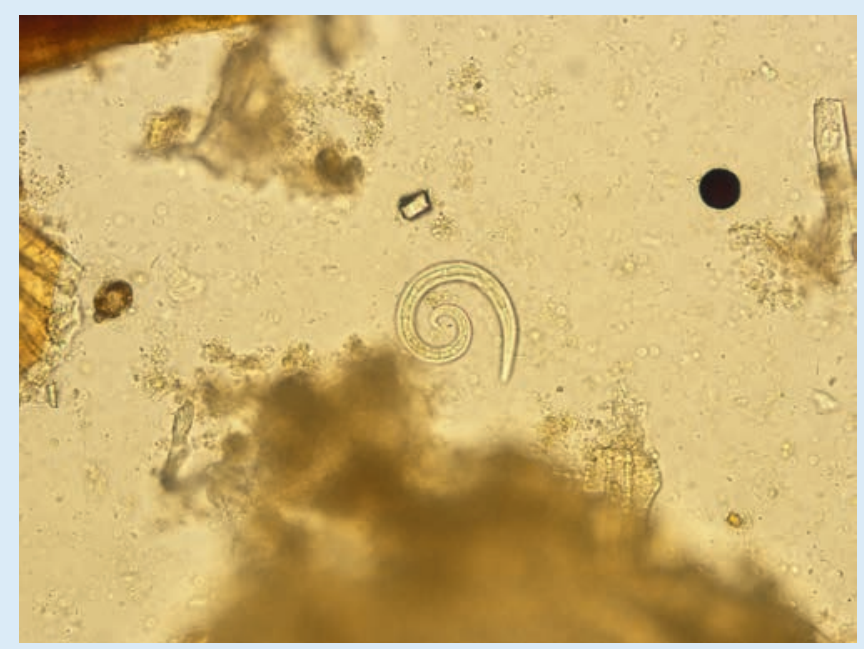

Abb. 1 Larve von Angiostrongylus vasorum. gerte Gerinnungszeit und Blutungen in die verschiedensten Organe)

Eine disseminierte Gerinnung führt zum Verbrauch verschiedener Gerinnungsfaktoren. Betroffen sind sowohl das primäre Gerinnungssystem durch eine massive Thrombozytopenie als auch das sekundäre durch den Verbrauch von Faktor V, Prothrombin und Thromboplastin. Die Infektion im rechten Herzen kann zu einer Rechtsherzsymptomatik führen, bei massivem Befall kann es in seltenen Fällen zu plötzlichen Todesfällen kommen.

Chronische Fälle zeichnen sich durch die Folgen der Besiedlung des rechten Herzens mit Überdruck in der Pulmonalarterie aus. Außerdem kann eine chronische Infektion zu Gewichtsverlust führen. Seltene Symptome werden durch Larven verursacht, die in andere Gewebe gelangen. Hierzu gehören neurologische Ausfallserscheinungen mit Paralysen oder Zeichen von Schmerzen.

Im Blutbild können verschiedene unspezifische, meist milde Veränderungen festgestellt werden, wie z.B. eine regenerative Anämie, eine Eosinophilie sowie eine Thrombozytopenie. Veränderungen verschiedener Gerinnungsparameter wie PT und PTT sowie der Faktoren VIII und V können auftreten.

\section{Differenzialdiagnosen}

Zwingerhusten oder eine Infektion mit Crenosoma vulpis können eine ähnliche Symptomatik hervorrufen. $\mathrm{Zu}$ einem trockenen, harten Husten können auch Rechtsherzinsuffizienzen („Herzhusten“) oder allergische Erkrankungen führen.

\section{Diagnostik}

Als Standarduntersuchung gilt bisher das Auswanderungsverfahren nach Baermann-Wetzel. Dabei sollte eine möglichst frische Kotprobe zur Untersuchung gelangen, die auch nicht mit Erdnematoden kontaminiert ist. Die Sensitivität des Auswanderungsverfahrens liegt zwischen 
$51 \%$ und $65 \%$ und ist davon abhängig, ob eine Einzel- oder eine Sammelkotprobe untersucht wurde (bei einer Sammelkotprobe ist die Sensitivität höher). Die Spezifität liegt bei $98 \%$. Da die Larven ( $\triangleright$ Abb.1) intermittierend ausgeschieden werden, sollte diese Untersuchung nach negativem Ergebnis bei klinischer Symptomatik wiederholt werden.

Die Larven können auch in Trachealoder Bronchialspülproben mikroskopisch nachgewiesen werden. Das Sediment der Spülprobe kann mit einer Zellfärbung angefärbt werden, um die Wurmlarven gut darzustellen.

Wegen der fraglichen Sensitivität des Auswanderungsverfahrens wurde von Schnyder et al. (2011) ein ELISA als sensitives Verfahren zum Nachweis des im Blut zirkulierenden Antigens entwickelt. Die Autoren geben für diesen Test eine Sensitivität von 96\% und eine Spezifität von $94 \%$ an (Schnyder et al. 2011). Inzwischen ist auch ein praxisinterner Antigentest auf dem Markt, der auf Immunografie beruht.

Als weiteres Verfahren wurde eine Realtime-PCR entwickelt, die sowohl aus Blut und Serum als auch aus Kot funktioniert. Hier liegen aber noch zu wenige Ergebnisse für eine abschließende Bewertung vor.

\section{Therapie}

Nach Angaben der ESCCAP stehen zur Therapie von Angiostrongylus vasorum effektive Anthelmintika aus der Gruppe der makrozyklischen Laktone (Moxidectin, Milbemycinoxime) oder der Benzimidazole (Fenbendazol) zur Verfügung.

Mögliche Behandlungsschemata sind:

- Moxidectin/Imidacloprid (Advocate ${ }^{\circledR}$, Bayer Vital GmbH): 1-malige Spot-OnBehandlung (2,5 mg/kg KM Moxidectin und $10 \mathrm{mg} / \mathrm{kg} \mathrm{KM}$ Imidacloprid), evtl. Wiederholung nach 4 Wochen

- Milbemycinoxim (Milbactor, CEVA Tiergesundheit GmbH; Milbemax ${ }^{\circledR}$, Elanco; Milprazon $^{\circledR}$, TAD Pharma GmbH; Milpro, Virbac Tierarzneimittel $\mathrm{GmbH}$ ):

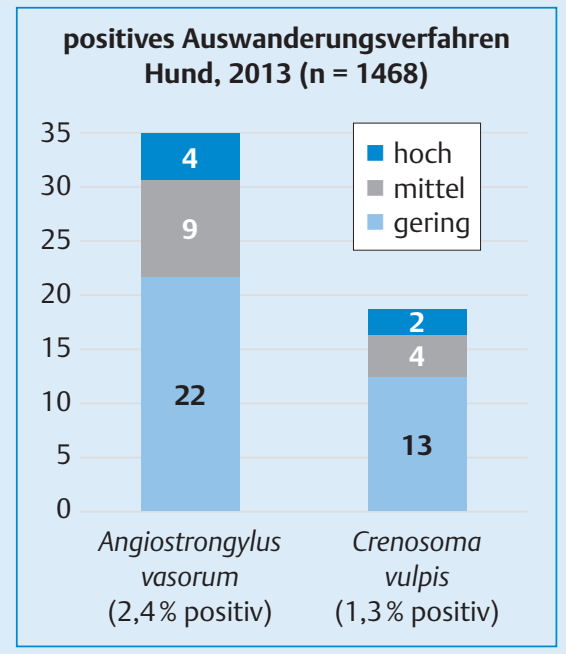

Abb. 2 Ergebnisse des Auswanderungsverfahrens aus Routineeinsendungen im Jahr 2013.

4-malige perorale Behandlung (0,5 mg/ kg KM) im wöchentlichen Abstand

- Fenbendazol (Panacur ${ }^{\circledR}$, MSD Tiergesundheit): täglich perorale Verabreichung (50 mg/kg KM) über 3 Wochen

Bei schweren klinischen Symptomen ist die Gabe von Antibiotika und Glukokortikoiden zu erwägen.

\section{Prävalenz}

Man ist immer davon ausgegangen, dass Angiostrongylus vasorum nicht oder nur lokal vereinzelt enzootisch in Deutschland vorkommt. So wurden in Untersuchungen zur Jahrtausendwende Prävalenzen von 0,1\% für Angiostrongylus vasorum und 0,3\% für Crenosoma vulpis angegeben (Barutzki und Schaper 2003). Gezielte Untersuchungen von Barutzki und Schaper (2009) in Deutschland an Hunden mit einer ungeklärten Lungensymptomatik erbrachten allerdings Prävalenzen von 7,4\% bei Angiostrongylus vasorum und 6,0\% bei Crenosoma vulpis.

\section{Ergebnisse aus Routineeinsendungen}

Kotproben aus Routineeinsendungen wurden mittels Auswanderungsverfahren nach Baermann-Wetzel untersucht, ein Vorbericht zur Klinik war nicht vorhanden. positives Auswanderungsverfahren Hund, 2014 ( $n=1938)$

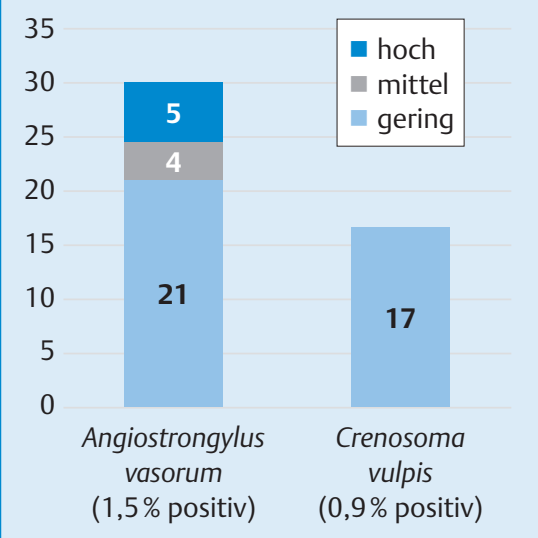

Abb. 3 Ergebnisse des Auswanderungsverfahrens aus Routineeinsendungen im Jahr 2014.

Die Daten aus den Jahren 2013 ( $>$ Abb. 2) und 2014 ( Abb.3) zeigen, dass man auch in Deutschland bei ungeklärter Lungensymptomatik immer an einen Befall mit Angiostrongylus vasorum denken muss. Die geringere Nachweisrate im Vergleich zu den Daten von Barutzki und Schaper aus 2009 dürfte auf die relativ ungezielte Untersuchung zurückzuführen sein.

Andere Daten aus einer Studie von Maksimov (Zeitraum 2003-2013) erbrachten Prävalenzen von 2,1\% bei Angiostrongylus vasorum und $1,8 \%$. bei Crenosoma vulpis. In dieser Studie wurden auch die Verteilungsraten in Deutschland ausgewertet, hierbei wurden vor allem im Südwesten und am Rhein entlang positive Befunde erhoben. Außerdem wurden in den Ballungsgebieten um Berlin und München positive Ergebnisse erzielt.

Online zu finden unter

http://dx.doi.org/10.1055/s-0035-1550138

\section{Literatur}

Literatur beim Verfasser

Dr. Anton Heusinger

Laboklin GmbH \& Co. KG

Steubenstr. 4

97688 Bad Kissingen 